\title{
The Application of Predictor Feedback in Designing a Preview Controller for Discrete-Time Systems with Input Delay
}

\author{
Fucheng Liao, ${ }^{1}$ Yonglong Liao, ${ }^{1}$ and Jiamei Deng ${ }^{2}$ \\ ${ }^{1}$ School of Mathematics and Physics, University of Science and Technology Beijing, Beijing 100083, China \\ ${ }^{2}$ Leeds Sustainability Institute, Leeds Beckett University, Leeds LS2 9EN, UK \\ Correspondence should be addressed to Fucheng Liao; fcliao@ustb.edu.cn
}

Received 15 April 2016; Revised 19 July 2016; Accepted 25 July 2016

Academic Editor: Jean J. Loiseau

Copyright (c) 2016 Fucheng Liao et al. This is an open access article distributed under the Creative Commons Attribution License, which permits unrestricted use, distribution, and reproduction in any medium, provided the original work is properly cited.

\begin{abstract}
This paper presents a method for designing a type one servomechanism for a discrete-time linear system with input delay subject to a previewable desired output and a nonmeasurable constant disturbance. The tracking problem of a delay system is transformed into a regulation problem of a delay-free system via constructing an augmented error system and a variable substitution. A controller is obtained with delay compensation and preview compensation based on preview control theory and the predictor method. When the state vector is not directly measurable, a full-dimensional observer is offered. The effectiveness of the design method is demonstrated by numerical simulations.
\end{abstract}

\section{Introduction}

Preview control is one of the approaches available for producing a good performance by utilizing future information of the reference signal in the controller. Lots of work on the preview control problem have already been done. One of the early contributions was delineated in [1], in which a statefeedback controller with preview compensation is derived. Furthermore, Katayama et al. used the linear quadratic integral method to study preview control in both discretetime and continuous-time systems [2,3]. Servomechanisms with integral of tracking error and preview compensation are derived. Liao et al. presented a design method of an optimal preview servomechanism for discrete-time systems in multirate sampled systems [4]. Liao et al. studied the preview control problem of discrete-time descriptor systems and produced a preview controller for the systems [5]. Recently, infinite-horizon $H_{\infty}$ state-feedback preview tracking control of retarded state-multiplicative stochastic systems was investigated [6]. In many practical designs, preview control draws considerable favor from engineering researchers, for example, robots walking [7-9], motorcycle performance $[10,11]$, and protection against earthquakes [12].

During the past several decades, there have been very rich research achievements in the area of control systems with time delay. An early control method for systems with time delay is the Smith predictor [13], which overcomes the dead time effectively by adding a predictor and a compensator in the controller. Furukawa and Shimemura improved the Smith predictor and offered a new control strategy called "predictive control" [14]. The control module consists of a predictor, an observer, and a controller. Thus, the range of the controller's application is expanded. Manitius and Olbrot studied the problems of finite spectrum assignment (FSA) of delay systems [15]. The resulting controllers involve integral compensation of the input delay and stabilize the closed-loop systems successfully. The FSA method has been a popular tool in dealing with delay systems. Stable conditions and numerical integral rules were given in [16-18] because the introduction of integral compensation in the controller might lead to closed-loop systems becoming unstable in numerical calculation [19]. Léonard and Abba studied FSA integral control robustness with respect to prediction time uncertainty for an unstable system [20]. The optimal regulation problem was studied for systems with input delay and designed optimal feedback controllers by using the duality principle and the maximum principle [21-23]. The design process was simplified by introducing a quadratic performance index with corresponding input delay in [24]. A nested predictor was established to effectively compensate for the time delay for 
linear systems with both state and input delays [25, 26]. Robust control and function control strategies were given, respectively, for discrete-time delay systems in [27, 28]. More recently, the optimal control problem was studied for systems with both state and input delays based on the method of letting the preview length go to zero [29]. Optimal tracking controllers for the delay systems are obtained.

For discrete-time linear systems, if the input vector has a time delay, then it is necessary to reconsider the design of the preview controller. The input delay system was transformed into a delay-free system by using the discrete lifting technique in $[30,31]$. The researchers then obtained a preview controller based on preview control theory. However, it was pointed out that the discrete lifting technique may lead to "dimension disaster," especially for systems with large delays [23, 32]. Therefore, this paper further studies the preview control problem of linear systems with input delay. A controller with delay compensation and preview compensation will be developed by using the method of predictor feedback.

This paper is organized as follows. An introduction is given in Section 1. Section 2 is a formulation of the problem and gives some basic assumptions. Section 3 uses the preview control method to construct an augmented error system. Based on Section 3, Section 4 derives a preview controller for the original system. Section 5 constructs a full-dimensional observer. And a brief conclusion is drawn in Section 6 .

\section{Problem Formulation and Basic Assumptions}

Consider a discrete-time system with input delay as follows:

$$
\begin{aligned}
x(k+1) & =A x(k)+B u(k-f)+E w(k), \\
y(k) & =C x(k),
\end{aligned}
$$

where $x(k) \in R^{n}$ is the state vector, $u(k) \in R^{r}$ is the input vector, $y(k) \in R^{p}$ is the output vector, and $w(k) \in R^{q}$ is the nonmeasurable constant disturbance. The positive integer $f$ represents a constant input delay of the system. $A \in R^{n \times n}$, $B \in R^{n \times r}, C \in R^{p \times n}$, and $E \in R^{n \times q}$ are constant matrices. The vectors $u(-f), u(-f+1), \ldots, u(0)$ are initial inputs, and the vector $x(0)=x_{0}$ is the initial state. All the initial vectors are known.

Let $r(k) \in R^{p}$ be the reference signal.

First, we give the following two basic assumptions:

(A1) Let the pairs $(A, B)$ be stabilizable, let $(C, A)$ be detectable, and let the following conditions hold:

$$
\operatorname{rank}\left[\begin{array}{cc}
A & B \\
C & 0
\end{array}\right]=n+p \quad(\text { full row rank })
$$

(A2) Let the reference signal $r(k)$ be previewable, and its preview length is $N_{r}$. Namely, at the present time $k$, the present value $r(k)$ as well as the $N_{r}$ future values $r(k+1), \ldots, r\left(k+N_{r}\right)$ is available. The future values of the reference signal beyond time $k+N_{r}$ are approximated by $r\left(k+N_{r}\right)$; namely, $r(k+l)=r\left(k+N_{r}\right)$ $\left(l \geq N_{r}+1\right)$. The reference signal $r(k)$ satisfies

$$
\lim _{k \rightarrow \infty} r(k)=\bar{r},
$$

where $\bar{r}$ is a constant vector. This implies that $r(k)$ reaches a steady state.

Furthermore, let $e(k)$ be the error signal defined as the subtraction of $y(k)$ and $r(k)$; that is,

$$
e(k)=y(k)-r(k) .
$$

The purpose of this paper is to design a controller with preview compensation such that the output $y(k)$ of the closed-loop system of (1) tracks the reference signal $r(k)$ without any static error in the presence of disturbance $w(k)$; namely,

$$
\lim _{k \rightarrow \infty} e(k)=0 .
$$

The optimal control method is applied to achieve the goal. The performance index of (1) can be defined as

$$
J=\sum_{k=1}^{\infty}\left[e^{T}(k) Q_{e} e(k)+\Delta u^{T}(k-f) H \Delta u(k-f)\right],
$$

where $Q_{e} \in R^{p \times p}$ and $H \in R^{r \times r}$ are positive definite weight matrices. $\Delta$ is the first-order backward difference operator; that is,

$$
\Delta u(k)=u(k)-u(k-1) .
$$

Notice that, as used in [2], the performance index (6) uses the input vector's difference $\Delta u(k)$ rather than $u(k)$. Introducing the input vector's difference into the performance index can make the closed-loop system contain an integrator, which may help the system to eliminate static error $[2,33]$.

\section{Derivation of the Augmented Error System}

The basic method of designing a preview controller for (1) is that an augmented error system is constructed firstly, then a controller is derived for the augmented error system by using optimal preview theory and predictor feedback, and finally the controller for the original system is obtained.

Using $\Delta$ on both sides of the first equation of (1), the following will stand:

$$
\Delta x(k+1)=A \Delta x(k)+B \Delta u(k-f),
$$

where it is obvious that the disturbance vector's difference $\Delta w(k)$ does not appear because the disturbance is a constant.

Using $\Delta$ on $e(k+1)$ and noticing $e(k+1)=C x(k+1)-$ $r(k+1)$, the following will be obtained:

$$
\Delta e(k+1)=C \Delta x(k+1)-\Delta r(k+1) .
$$

Since $\Delta e(k+1)=e(k+1)-e(k)$, it can be seen from (8) and (9) that the error signal satisfies

$$
\begin{aligned}
e(k+1)= & e(k)+C A \Delta x(k)+C B \Delta u(k-f) \\
& -\Delta r(k+1) .
\end{aligned}
$$


Combining (8) and (10) yields

$$
\begin{aligned}
X(k+1) & =\bar{A} X(k)+\bar{B} \Delta u(k-f)+\bar{D} \Delta r(k+1), \\
e(k) & =\bar{C} X(k),
\end{aligned}
$$

where

$$
\begin{aligned}
X(k) & =\left[\begin{array}{c}
e(k) \\
\Delta x(k)
\end{array}\right], \\
\bar{A} & =\left[\begin{array}{cc}
I_{p} & C A \\
0 & A
\end{array}\right], \\
\bar{B} & =\left[\begin{array}{c}
C B \\
B
\end{array}\right], \\
\bar{C} & =\left[\begin{array}{ll}
I_{p} & 0
\end{array}\right], \\
\bar{D} & =\left[\begin{array}{c}
-I_{p} \\
0
\end{array}\right] .
\end{aligned}
$$

Equation (11) is called the augmented error system of (1). It is appropriate to take $e(k)=\bar{C} X(k)$ as the output of (11), because the output of (1) is $y(k)$ and the reference signal $r(k)$ is previewable.

Correspondingly, in terms of the augmented state vector $X(k)$, the performance index (6) can be expressed as

$$
J=\sum_{k=1}^{\infty}\left[X^{T}(k) Q X(k)+\Delta u^{T}(k-f) H \Delta u(k-f)\right],
$$

where

$$
Q=\left[\begin{array}{cc}
Q_{e} & 0 \\
0 & 0
\end{array}\right]
$$

If a controller $\Delta u(k)$ can be derived such that the performance index (13) minimum is subject to the dynamic constraint (11), then it is easy to get $\lim _{k \rightarrow \infty} X(k)=0$, and immediately the conclusion $\lim _{k \rightarrow \infty} e(k)=0$ holds. Furthermore, the input $u(k)$ can be solved from $\Delta u(k)$. And, thus, the purpose is achieved. This is a standard optimal preview control problem.

\section{Main Results and Their Proofs}

Let us introduce a new input vector

$$
v(k)=\Delta u(k-f) .
$$

Substituting (15) into (11) and (13), respectively, the following stands:

$$
\begin{aligned}
X(k+1) & =\bar{A} X(k)+\bar{B} v(k)+\bar{D} \Delta r(k+1), \\
e(k) & =\bar{C} X(k), \\
J & =\sum_{k=1}^{\infty}\left[X^{T}(k) Q X(k)+v^{T}(k) H v(k)\right] .
\end{aligned}
$$

Obviously, (16) is a delay-free system and the performance criterion (17) has a normal form. Furthermore, it is known from (A2) that the reference signal $r(k)$ is previewable in the sense that the future value $\Delta r(l)(k \leq l \leq k+$ $N_{r}$ ) is available at each instant of time $k$. This is a preview control problem in which the system is described as (16), the quadratic performance index is described as (17), and $\Delta r(k)$ is previewable. The following theorem will stand based on the results of [2].

Theorem 1. If (A1) and (A2) hold and $Q_{e}$ is positive definite, then the preview controller of (16) that minimizes criterion (17) is given by

$$
v(k)=-G_{X} X(k)-\sum_{l=1}^{N_{r}} G_{d}(l) \Delta r(k+l),
$$

where

$$
\begin{aligned}
G_{X} & =\left[H+\bar{B}^{T} P \bar{B}\right]^{-1} \bar{B}^{T} P \bar{A}, \\
G_{d}(1) & =-\left[H+\bar{B}^{T} P \bar{B}^{-1} \bar{B}^{T} P\left[\begin{array}{c}
I_{p} \\
0
\end{array}\right],\right. \\
G_{d}(l) & =\left[H+\bar{B}^{T} P \bar{B}\right]^{-1} \bar{B}^{T} \widetilde{X}(l-1), \quad l=2, \ldots, N_{r},
\end{aligned}
$$

where $P \in R^{(p+n) \times(p+n)}$ is the positive semidefinite solution of the algebraic Riccati equation:

$$
P=\bar{A}^{T} P \bar{A}-\bar{A}^{T} P \bar{B}\left[H+\bar{B}^{T} P \bar{B}^{-1} \bar{B}^{T} P \bar{A}+Q .\right.
$$

Furthermore, the matrices $\widetilde{X}(l) \in R^{(n+p) \times p}$ are given by

$$
\begin{aligned}
& \widetilde{X}(1)=-\bar{A}_{c}^{T} P\left[\begin{array}{c}
I_{p} \\
0
\end{array}\right] ; \\
& \widetilde{X}(l)=\bar{A}_{c}^{T} \widetilde{X}(l-1), \quad l=2, \ldots, N_{r},
\end{aligned}
$$

where $\bar{A}_{c}$ is the closed-loop matrix defined by

$$
\bar{A}_{c}=\bar{A}-\bar{B}\left[H+\bar{B}^{T} P \bar{B}\right]^{-1} \bar{B}^{T} P \bar{A} .
$$

Remark 2. The future reference signal value $\Delta r(k+l)(l=$ $\left.1, \ldots, N_{r}\right)$ appearing in $\sum_{l=1}^{N_{r}} G_{d}(l) \Delta r(k+l)$ acts as a preview compensation in the controller.

The preview controller for the augmented error system (11) can be derived from Theorem 1 by the following method.

Combining (15) and (18), the following equation will be obtained:

$$
\Delta u(k-f)=-G_{X} X(k)-\sum_{l=1}^{N_{r}} G_{d}(l) \Delta r(k+l) .
$$

Replacing $k-f$ with $k$ leads to

$$
\Delta u(k)=-G_{X} X(k+f)-\sum_{l=1}^{N_{r}} G_{d}(l) \Delta r(k+f+l) .
$$


In (24), the current control input $\Delta u(k)$ uses the future state vector $X(k+f)$. It is necessary to predict the future state vector to ensure that the controller is executable. Using the stepwise recurrence technique, we solve the state equation of (11):

$$
X(k+1)=\bar{A} X(k)+\bar{B} \Delta u(k-f)+\bar{D} \Delta r(k+1),
$$

and obtain the future value of the state vector

$$
\begin{aligned}
X(k+f)= & \bar{A}^{f} X(k)+\sum_{l=0}^{f-1} \bar{A}^{(f-1-l)} \bar{B} \Delta u(k+l-f) \\
& +\sum_{l=0}^{f-1} \bar{A}^{(f-1-l)} \bar{D} \Delta r(k+1+l) .
\end{aligned}
$$

The future value $X(k+f)$ is predicted by (26). According to Assumption (A2), for the preview length $N_{r} \geq f$, the vectors $\Delta r(k+1+l)$ are available at $l=0, \ldots, f-1$; for the preview length $N_{r}<f$, the vectors $\Delta r(k+1+l)$ are available at $l=0, \ldots, N_{r}-1$; and $\Delta r(k+1+l)=0$ at $l=N_{r}, \ldots, f-1$. Obviously, the values in (26) are all available. Equation (26) indicates that the future state vector $X(k+f)$ is determined by the current state vector $X(k)$, the past control input $\Delta u(k+l-f)(l=0, \ldots, f-1)$, and the future reference signal's difference $\Delta r(k+1+l)(l=0, \ldots, f-1)$. The predictor method we used above is a generalization of the predictor feedback method [14].

Substituting (26) into (24), the feedback control law of the augmented error system (11) can be obtained as follows:

$$
\begin{aligned}
\Delta u(k)= & -G_{X} \bar{A}^{f} X(k) \\
& -G_{X} \sum_{l=0}^{f-1} \bar{A}^{(f-1-l)} \bar{B} \Delta u(k+l-f) \\
& -G_{X} \sum_{l=0}^{f-1} \bar{A}^{(f-1-l)} \bar{D} \Delta r(k+1+l) \\
& -\sum_{l=1}^{N_{r}} G_{d}(l) \Delta r(k+f+l) .
\end{aligned}
$$

Considering the last term of $\sum_{l=1}^{N_{r}} G_{d}(l) \Delta r(k+f+l)$ in (27), if $k+f+l>k+N_{r}$, let $\Delta r(k+f+l)=0$. It is obvious that (27) is an executable controller of (11) since all of the parts in (27) are known.

Let us derive a preview controller of (1). First, let

$$
G_{X} \bar{A}^{f}=\left[\begin{array}{ll}
G_{e} & G_{x}
\end{array}\right]
$$

where $G_{e} \in R^{r \times p}$ and $G_{x} \in R^{r \times n}$; then, (27) can be rewritten as

$$
\begin{aligned}
\Delta u(k)= & -G_{e} e(k)-G_{x} \Delta x(k) \\
& -G_{X} \sum_{l=0}^{f-1} \bar{A}^{(f-1-l)} \bar{B} \Delta u(k+l-f) \\
& -G_{X} \sum_{l=0}^{f-1} \bar{A}^{(f-1-l)} \bar{D} \Delta r(k+1+l) \\
& -\sum_{l=1}^{N_{r}} G_{d}(l) \Delta r(k+f+l) .
\end{aligned}
$$

It is assumed that the initial values of system (1) and the reference signal are zeros; namely, for $k=-f,-f+1, \ldots, 0$, the vectors $x(k)=0, y(k)=r(k)=0$, and $u(k)=0$. Then, the following result from (29) will be obtained:

$$
\begin{aligned}
u(k)= & -G_{e} \sum_{j=1}^{k} e(j)-G_{x} x(k) \\
& -G_{X} \sum_{l=0}^{f-1} \bar{A}^{(f-1-l)} \bar{B} u(k+l-f) \\
& -G_{X} \sum_{l=0}^{f-1} \bar{A}^{(f-1-l)} \bar{D} r(k+1+l) \\
& -\sum_{l=1}^{N_{r}} G_{d}(l) r(k+f+l) .
\end{aligned}
$$

Thus, a preview control theory for system (1) will be obtained as follows.

Theorem 3. Let (A1) and (A2) hold, let $Q_{e}$ be positive definite, and let the performance index be defined as (8). Assume that $x(k)=0, y(k)=r(k)=0$, and $u(k)=0$ for $k=-f,-f+$ $1, \ldots, 0$. Then, the preview controller of (1) is given by

$$
u(k)=-G_{e} \sum_{j=1}^{k} e(j)-G_{x} x(k)-f_{1}(k)-f_{2}(k),
$$

where

$$
\begin{aligned}
f_{1}(k)= & G_{X} \sum_{l=0}^{f-1} \bar{A}^{(f-1-l)} \bar{B} u(k+l-f), \\
f_{2}(k)= & G_{X} \sum_{l=0}^{f-1} \bar{A}^{(f-1-l)} \bar{D} r(k+1+l) \\
& +\sum_{l=1}^{N_{r}} G_{d}(l) r(k+f+l),
\end{aligned}
$$

$G_{e}$ and $G_{x}$ are determined by (28), and $G_{X}$ and $G_{d}$ are given by Theorem 1 . 
Remark 4. At each time $k$, for the general term $r(k+f+l)$ in $\sum_{l=1}^{N_{r}} G_{d}(l) r(k+f+l)$, if $f+l \leq N_{r}$, then the real value of $r(k+f+l)$ is taken; if $f+l>N_{r}$, then $r(k+f+l)=r\left(k+N_{r}\right)$ is taken. This is determined by Assumption (A2).

Remark 5. It is easy to see that the preview controller (31) is composed of four terms. The first one $-G_{e} \sum_{j=1}^{k} e(j)$ is the accumulation of the tracking error, which ensures that the output of the closed-loop system tracks the reference signal without static error. The second one $-G_{x} x(k)$ is the state feedback. The third one $-f_{1}(k)$ is the compensation of the input delay. The last one $-f_{2}(k)$ is the preview compensation of the reference signal.

According to the character of the reference signal considered here, the abovementioned design method for the preview controller is applicable to some irregular reference signals which cannot be modeled by the dynamic system's outputs.

Now, let us apply present theory to an air slider linear motor.

Example 6 (see [34]). The dynamic equation of the motor is described as follows:

$$
\begin{aligned}
{\left[\begin{array}{c}
\dot{x}_{p}(t) \\
\dot{x}_{v}(t)
\end{array}\right]=} & {\left[\begin{array}{cc}
0 & 1 \\
0 & -\frac{D}{M}
\end{array}\right]\left[\begin{array}{l}
x_{p}(t) \\
x_{v}(t)
\end{array}\right]+\left[\begin{array}{c}
0 \\
\frac{K_{F}}{M}
\end{array}\right] u(t-\tau) } \\
& +\left[\begin{array}{c}
0 \\
-\frac{1}{M}
\end{array}\right] d(t),
\end{aligned}
$$

where $x_{p}(t)$ is place, $x_{v}(t)$ is velocity, $u(t)$ is current input, $d(t)$ is constant disturbance signal, $D$ is friction factor, $M$ is the mass of movable part, $K_{F}$ is propulsive force coefficient, and $\tau$ is input delay. The motor's parameters are $K_{F}=2.3$ (N/A), $M=1.82(\mathrm{~kg})$, and $D=3.48(\mathrm{~kg} / \mathrm{s})$, and $\tau$ could be taken as $0.10(\mathrm{~s}), 0.20(\mathrm{~s})$, and $0.30(\mathrm{~s})$, respectively.

Let

$$
x(t)=\left[\begin{array}{l}
x_{p}(t) \\
x_{v}(t)
\end{array}\right]
$$

then, (33) can be rewritten as

$$
\dot{x}(t)=A_{0} x(t)+B_{0} u(t-\tau)+E_{0} w(t) .
$$

The place of $x_{p}(t)$ is the output vector, and it can be described as

$$
y(t)=C x(t),
$$

where

$$
\begin{aligned}
A_{0} & =\left[\begin{array}{cc}
0 & 1 \\
0 & -\frac{D}{M}
\end{array}\right], \\
B_{0} & =\left[\begin{array}{c}
0 \\
\frac{K_{F}}{M}
\end{array}\right], \\
E_{0} & =\left[\begin{array}{c}
0 \\
-\frac{1}{M}
\end{array}\right], \\
C & =\left[\begin{array}{ll}
1 & 0
\end{array}\right] .
\end{aligned}
$$

Taking sampling period $T=0.01(\mathrm{~s})$, a discretization system is obtained:

$$
\begin{aligned}
x[(k+1) T] & =A x(k T)+B u[(k-f) T]+E w(k T), \\
y(k T) & =C x(k T),
\end{aligned}
$$

where

$$
\begin{aligned}
A & =\left[\begin{array}{ll}
1.0 & 0.009865 \\
0.0 & 0.913179
\end{array}\right], \\
B & =\left[\begin{array}{c}
0.0 \\
0.017804
\end{array}\right], \\
E & =\left[\begin{array}{c}
0.0 \\
-0.549451
\end{array}\right] .
\end{aligned}
$$

The delay $f$ is determined by $\tau$. According to the value of $\tau$, we have $f=10, f=20$, and $f=30$, respectively.

The reference signal $r(t)$ is given as the following two types.

(1) Step Signal. Let the reference signal be

$$
r(t)= \begin{cases}0, & t<0.2, \\ 1, & t \geq 0.2\end{cases}
$$

which can be discretized into

$$
r(k T)= \begin{cases}0, & k<20, \\ 1, & k \geq 20 .\end{cases}
$$

(2) Fading Signal. Let the reference signal be

$$
r(t)=\frac{2}{5 t+0.90} \sin (5 \pi(t+0.90))
$$

which can be discretized into

$$
r(k)=\frac{2}{(5 k+90) T} \sin (5 \pi(k+90) T) .
$$




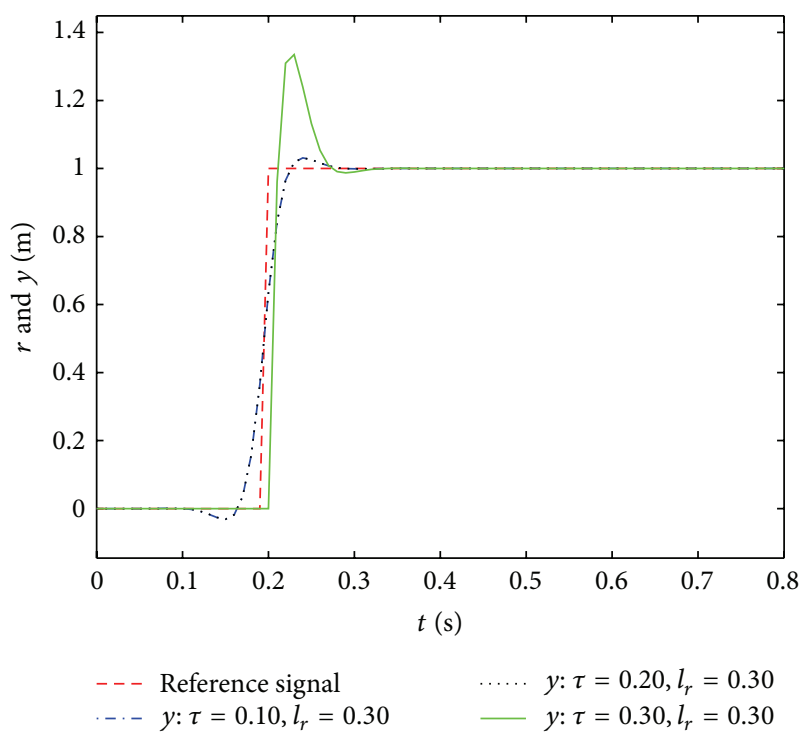

FIgURE 1: The output responses to the step signal with different delays.

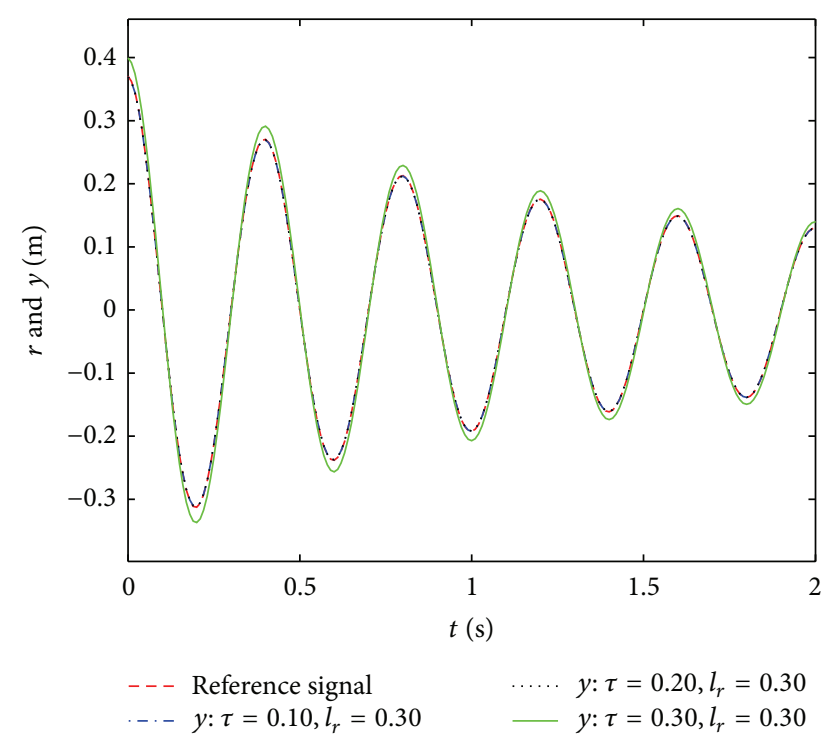

FIGURE 2: The output responses to the fading signal with different delays.

Through verification, $(A, B)$ is stabilizable, $(C, A)$ is detectable, and the matrix $\left[\begin{array}{ll}A & B \\ C & 0\end{array}\right]$ is of full row rank. Namely, Assumption (A1) is satisfied. We take the weight matrices $Q_{e}=1.0$ and $H=0.005$. By Theorem 3, there exists the preview controller described as (31) for (38).

Let the preview length of the reference signal be $l_{r}=$ 0.30 (s); that is, $N_{r}=30$. The output responses of the closedloop system are given as in Figures 1 and 2.

The output responses of the closed-loop system tracking to the step signal are shown in Figure 1 and those to the fading signal are shown in Figure 2. From Figures 1 and 2, it can be seen that the closed-loop's output can track the reference signal asymptotically with the controller (31). Comparing

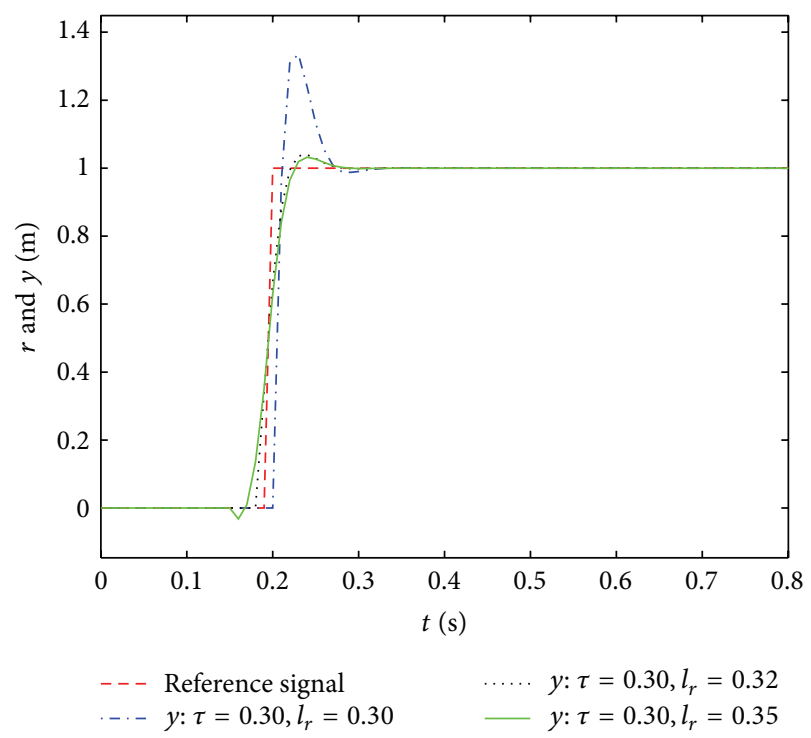

Figure 3: The output responses to the step signal with different preview lengths.

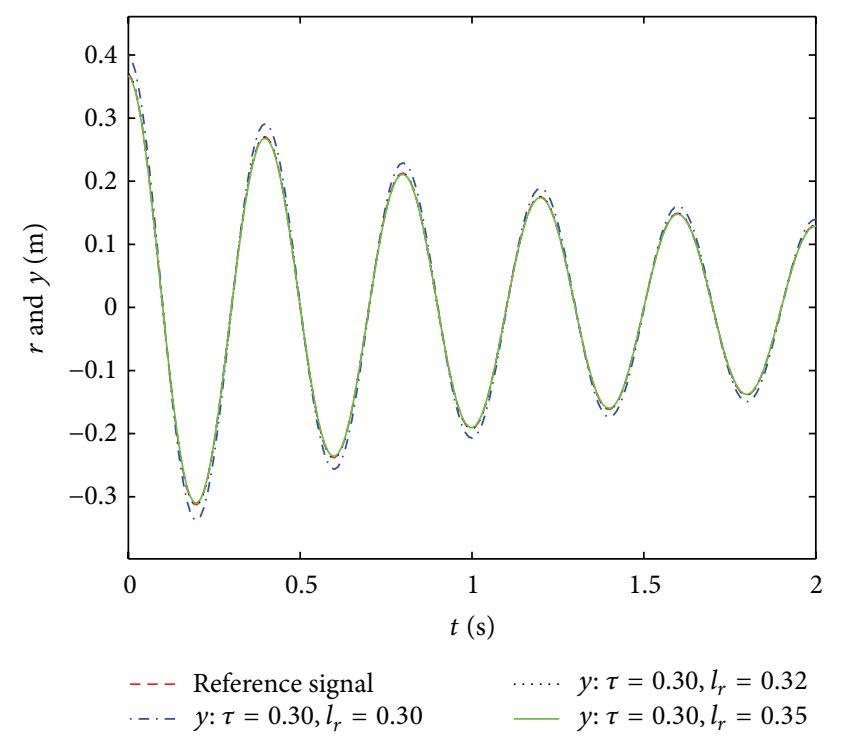

FIgURE 4: The output responses to the fading signal with different preview lengths.

with the output responses with different delays, it can be seen that under the same preview length the tracking performance will get worse when the delay is large.

When the preview length is equal to the input delay, the future value of the reference signal is only used to compensate the delay. In order to get a better performance, let us take a larger preview length. When the input delay $\tau=0.30(\mathrm{~s})$, the preview lengths $l_{r}=0.30(\mathrm{~s}), l_{r}=0.32(\mathrm{~s})$, and $l_{r}=0.35(\mathrm{~s})$ are taken; that is $N_{r}=30, N_{r}=32$, and $N_{r}=35$, respectively. The output responses of the closed-loop system are given as in Figures 3 and 4.

It can be seen from Figures 3 and 4 that the tracking performance is very good when the preview length is 
$N_{r}=35$, which indicates using the controller with preview compensation can reduce the tracking error and improve the tracking speed efficiently. On the other hand, a proper preview length can give a better response.

\section{Observer-Based Controller}

If the state vector $x(k)$ cannot be measured directly, then it is necessary to design an observer to obtain the estimate of the state vector.

Since $w(k)$ is constant but cannot be measured, we take it as a portion of the desired estimate vector. System (1) can be rewritten as

$$
\begin{aligned}
{\left[\begin{array}{c}
x(k+1) \\
w(k+1)
\end{array}\right] } & =\left[\begin{array}{cc}
A & E \\
0 & I_{q}
\end{array}\right]\left[\begin{array}{l}
x(k) \\
w(k)
\end{array}\right]+\left[\begin{array}{l}
B \\
0
\end{array}\right] u(k-f), \\
y(k) & =\left[\begin{array}{ll}
C & 0
\end{array}\right]\left[\begin{array}{l}
x(k) \\
w(k)
\end{array}\right] .
\end{aligned}
$$

Let $\widehat{x}(k)$ and $\widehat{w}(k)$ be the estimates of $x(k)$ and $w(k)$, respectively. Let $Y^{k-1}$ be the output values up to the $k-1$ step; namely, $Y^{k-1}=\{y(0), y(1), \ldots, y(k-1)\}$; let $\widehat{U}^{k-f}$ be the input values up to the $k-f$ step; namely, $\widehat{U}^{k-f}=$ $\{\widehat{u}(-f), \widehat{u}(-f+1), \ldots, \widehat{u}(-f+k)\}$. The input vector $\widehat{u}(i)(i=$ $-f,-f+1, \ldots,-f+k)$ here is obtained by replacing $x(k)$ by $\widehat{x}(k)$ in (31). Then, based on $Y^{k-1}$ and $\widehat{U}^{k-f}$, the full-order observer for the system of (44) is given by

$$
\begin{aligned}
{\left[\begin{array}{c}
\widehat{x}(k+1) \\
\widehat{w}(k+1)
\end{array}\right]=} & {\left[\begin{array}{ll}
A & E \\
0 & I_{q}
\end{array}\right]\left[\begin{array}{l}
\widehat{x}(k) \\
\widehat{w}(k)
\end{array}\right]+\left[\begin{array}{l}
B \\
0
\end{array}\right] \widehat{u}(k-f) } \\
& +\left[\begin{array}{l}
L_{x} \\
L_{w}
\end{array}\right](y(k)-\hat{y}(k)), \\
\widehat{y}(k)= & {\left[\begin{array}{ll}
C & 0
\end{array}\right]\left[\begin{array}{l}
\widehat{x}(k) \\
\widehat{w}(k)
\end{array}\right], }
\end{aligned}
$$

where $\hat{y}(k)$ is the output of the observer and $L_{x} \in R^{n \times p}$ and $L_{w} \in R^{q \times p}$ are constant gain matrices, which are determined so that the matrix

$$
\bar{A}_{L}=\left[\begin{array}{cc}
A-L_{x} C & E \\
-L_{w} C & I_{q}
\end{array}\right]
$$

is stable.

Lemma 7. Consider delay system (1). If $(C, A)$ is detectable and the matrix $\left[\begin{array}{cc}C & 0 \\ I-A & E\end{array}\right]$ is of full column rank, then there exist suitable gains $L_{x} \in R^{n \times p}$ and $L_{w} \in R^{q \times p}$ such that (45) serves as a full-order observer of (44).
Proof. According to the conclusions in [2], the pair $\left(\left[\begin{array}{ll}C & 0\end{array}\right],\left[\begin{array}{cc}A & E \\ 0 & I_{q}\end{array}\right]\right)$ is detectable if and only if $(C, A)$ is detectable and the matrix $\left[\begin{array}{cc}C & 0 \\ I-A & E\end{array}\right]$ is of full column rank. As a consequence, there exist suitable gains $L_{x} \in R^{n \times p}$ and $L_{w} \in R^{q \times p}$ such that $\bar{A}_{L}$ is stable under the conditions in Lemma 7. That is to say, the characteristic values of $\bar{A}_{L}$ are less than 1. Substituting the second formula of (45) into the first one and collecting the like terms, we get

$$
\begin{aligned}
{\left[\begin{array}{c}
\widehat{x}(k+1) \\
\widehat{w}(k+1)
\end{array}\right]=} & \bar{A}_{L}\left[\begin{array}{l}
\widehat{x}(k) \\
\widehat{w}(k)
\end{array}\right]+\left[\begin{array}{l}
B \\
0
\end{array}\right] \widehat{u}(k-f) \\
& +\left[\begin{array}{l}
L_{x} \\
L_{w}
\end{array}\right] y(k) .
\end{aligned}
$$

Let us define the subtraction of the actual state of (44) and the estimated state of (45)

$$
\left[\begin{array}{c}
\tilde{x}(k) \\
\widetilde{w}(k)
\end{array}\right]=\left[\begin{array}{l}
x(k) \\
w(k)
\end{array}\right]-\left[\begin{array}{l}
\widehat{x}(k) \\
\widehat{w}(k)
\end{array}\right]
$$

as estimation error. Notice that the input vector of (44) should be $\widehat{u}(k-f)$. Then, it is easy to get the estimation error dynamic equation as follows:

$$
\left[\begin{array}{c}
\widetilde{x}(k+1) \\
\widetilde{w}(k+1)
\end{array}\right]=\bar{A}_{L}\left[\begin{array}{c}
\widetilde{x}(k) \\
\widetilde{w}(k)
\end{array}\right] .
$$

Since the matrix $\bar{A}_{L}$ is stable, it follows that $\left[\begin{array}{c}\widetilde{x}(k) \\ \widetilde{w}(k)\end{array}\right]$ asymptotically converges to zero; namely,

$$
\lim _{t \rightarrow \infty}\left[\begin{array}{l}
\tilde{x}(k) \\
\widetilde{w}(k)
\end{array}\right]=0 .
$$

Thus, we have

$$
\lim _{k \rightarrow \infty}\left(\left[\begin{array}{l}
x(k) \\
w(k)
\end{array}\right]-\left[\begin{array}{l}
\widehat{x}(k) \\
\widehat{w}(k)
\end{array}\right]\right)=0
$$

which indicates that (45) can serve as a full-order observer of (44). This completes the proof.

From Lemma 7, it is known that $\widehat{x}(k)$ and $\widehat{w}(k)$ can serve as the reconstructed states of $x(k)$ and $w(k)$, respectively. Now, a preview control theorem with observer for system (1) is given as follows.

Theorem 8. If (A1) and (A2) hold, $Q_{e}$ is positive definite, the matrix $\left[\begin{array}{cc}C & 0 \\ I-A & E\end{array}\right]$ is of full column rank, and, for $k=-f,-f+$ $1, \ldots, 0$, the vectors $x(k)=0, y(k)=r(k)=0$, and $u(k)=0$, 


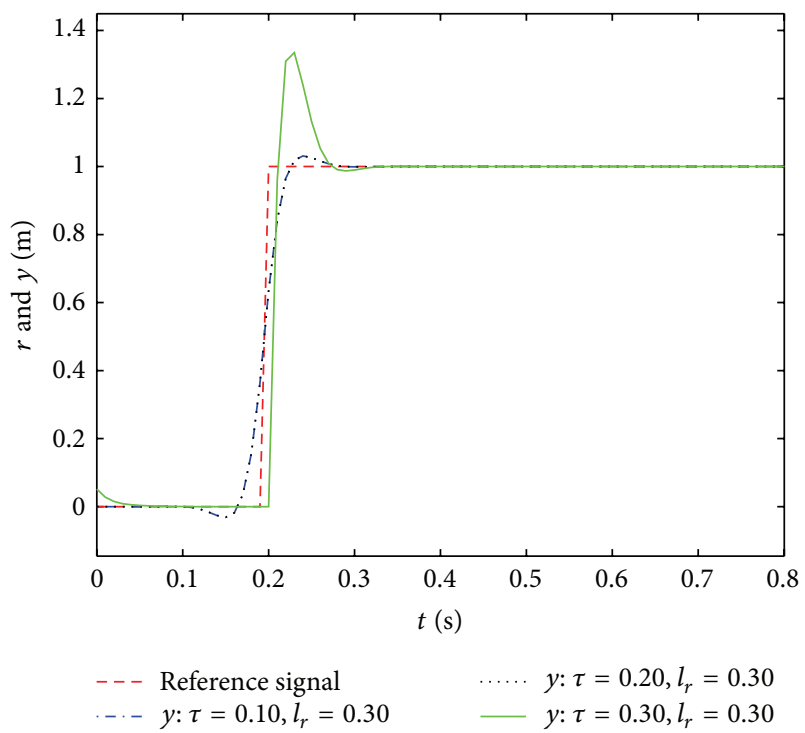

Figure 5: The output responses with state observer to the step signal.

then the closed-loop system of (1) with state observer is given by

$$
\begin{aligned}
x(k+1)= & A x(k)+B \widehat{u}(k-f)+E w(k), \\
y(k)= & C x(k), \\
e(k)= & y(k)-r(k), \\
{\left[\begin{array}{c}
\widehat{x}(k+1) \\
\widehat{w}(k+1)
\end{array}\right]=} & \bar{A}_{L}\left[\begin{array}{l}
\widehat{x}(k) \\
\widehat{w}(k)
\end{array}\right]+\left[\begin{array}{l}
B \\
0
\end{array}\right] \widehat{u}(k-f) \\
& +\left[\begin{array}{c}
L_{x} \\
L_{w}
\end{array}\right] y(k), \\
\widehat{u}(k)= & -G_{e} \sum_{j=1}^{k} e(j)-G_{x}\left[\begin{array}{ll}
I_{n} & 0
\end{array}\right]\left[\begin{array}{l}
\widehat{x}(k) \\
\widehat{w}(k)
\end{array}\right] \\
& -f_{1}(k)-f_{2}(k) .
\end{aligned}
$$

The gain matrices $L_{x} \in R^{n \times p}$ and $L_{w} \in R^{q \times p}$ are constant, which are determined such that the matrix $\bar{A}_{L}$ is stable. $f_{1}(k)$ and $f_{2}(k)$ have the following forms:

$$
\begin{aligned}
f_{1}(k)= & G_{X} \sum_{l=0}^{f-1} \bar{A}^{(f-1-l)} \bar{B} \hat{u}(k+l-f), \\
f_{2}(k)= & G_{X} \sum_{l=0}^{f-1} \bar{A}^{(f-1-l)} \bar{D} r(k+1+l) \\
& +\sum_{l=1}^{N_{r}} G_{d}(l) r(k+f+l),
\end{aligned}
$$

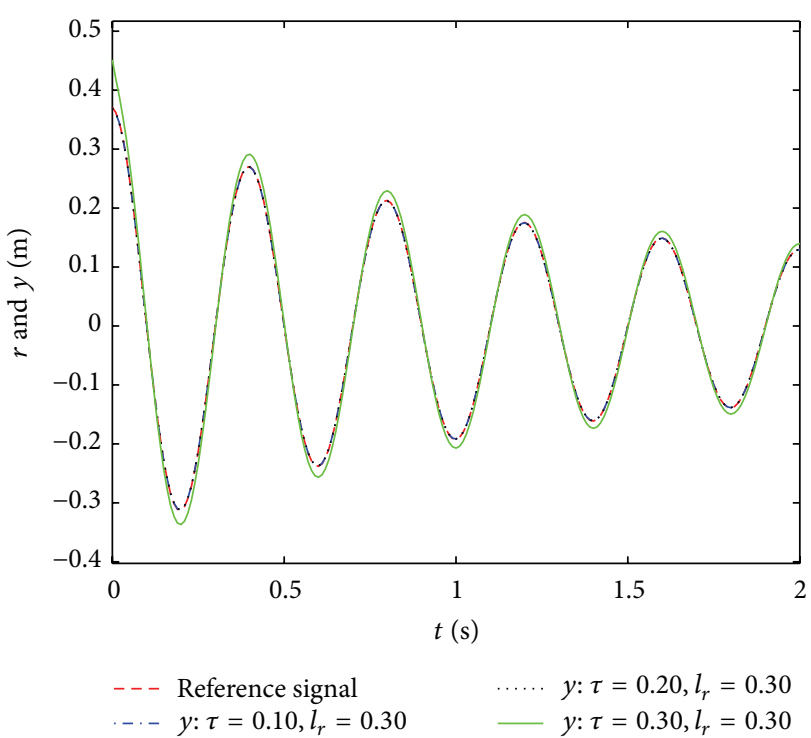

FIGURE 6: The output responses with state observer to the fading signal.

where $G_{e}$ and $G_{x}$ are determined by (28) and $G_{X}$ and $G_{d}$ are given by Theorem 1 .

Example 9. Consider the control system in Example 6. If the state vector cannot be measured directly, then the technique described in Theorem 8 is used to design a controller for the plant. The matrix $\left[\begin{array}{cc}C & 0 \\ I-A & E\end{array}\right]$ is of full column rank and therefore there exists a full-order observer with the form of (45). Let $L_{x}=\left[\begin{array}{ll}-288.14 & 144.77\end{array}\right]^{T}$ and $L_{w}=-22.13$ serve as the observer's gain matrices. The output responses are described in Figures 5 and 6.

Compared with the responses in Figures 1 and 2, the output responses in Figures 5 and 6 have a larger error in the initial phase; the tracking performances later are almost the same. The simulation results indicate the design methods for the preview controller with full-order observer are effective.

\section{Conclusion}

In this paper, a class of preview controller problems of discrete-time linear systems with input delay has been solved. A preview controller with delay compensation and preview compensation is derived. The difficulties of input delay are successfully overcome by predictor feedback. A design method of the full state observer is given when the state vector of the system cannot be measured directly. Numerical results show that the present methods are effective.

Further studies are needed on selecting a proper preview length according to the characteristics of the system, which is a complex problem but a significant one.

\section{Competing Interests}

The authors declare that they have no competing interests. 


\section{Acknowledgments}

The authors would like to thank the National Natural Science Foundation of China for their financial support under Grant no. 61174209.

\section{References}

[1] M. Hayase and K. Ichikawa, "Optimal servosystem utilizing future value of desired function," Transactions of the Society of Instrument and Control Engineers, vol. 5, no. 1, pp. 86-94, 1969.

[2] T. Katayama, T. Ohki, T. Inoue, and T. Kato, "Design of an optimal controller for a discrete-time system subject to previewable demand," International Journal of Control, vol. 41, no. 3, pp. 677-699, 1985.

[3] T. Katayama and T. Hirono, "Design of an optimal servomechanism with preview action and its dual problem," International Journal of Control, vol. 45, no. 2, pp. 407-420, 1987.

[4] F. Liao, K. Takaba, T. Katayama, and J. Katsuura, "Design of an optimal preview servomechanism for discrete-time systems in multirate setting," Dynamics of Continuous, Discrete, and Impulsive Systems Series B: Applications and Algorithms, vol. 10, no. 5, pp. 727-744, 2003.

[5] F. Liao, M. Cao, Z. Hu, and P. An, "Design of an optimal preview controller for linear discrete time causal descriptor systems," International Journal of Control, vol. 85, no. 10, pp. 1616-1624, 2012.

[6] E. Gershon and U. Shaked, " $H_{\infty}$ preview tracking control of retarded state-multiplicative stochastic systems," International Journal of Robust and Nonlinear Control, vol. 24, no. 15, pp. 21192135, 2014.

[7] S. Kajita, F. Kanehiro, K. Kaneko et al., "Biped walking pattern generation by using preview control of zero-moment point," in Proceedings of the IEEE International Conference on Robotics and Automation, vol. 2, pp. 1620-1626, Taipei, Taiwan, September 2003.

[8] S. Shimmyo, T. Sato, and K. Ohnishi, "Biped walking pattern generation by using preview control based on three-mass model," IEEE Transactions on Industrial Electronics, vol. 60, no. 11, pp. 5137-5147, 2013.

[9] S. Czarnetzki, S. Kerner, and O. Urbann, "Observer-based dynamic walking control for biped robots," Robotics and Autonomous Systems, vol. 57, no. 8, pp. 839-845, 2009.

[10] R. S. Sharp, "Optimal preview speed-tracking control for motorcycles," Multibody System Dynamics, vol. 18, no. 3, pp. 397-411, 2007.

[11] J. Marzbanrad, Y. Hojjat, H. Zohoor, and S. K. Nikravesh, "Optimal preview control design of an active suspension based on a full car model," Scientia Iranica, vol. 10, no. 1, pp. 23-36, 2003.

[12] J. Marzbanrad, G. Ahmadi, and R. Jha, "Optimal preview active control of structures during earthquakes," Engineering Structures, vol. 26, no. 10, pp. 1463-1471, 2004.

[13] O. J. M. Smith, “A controller to overcome dead time," Journal of Instrument Society of America, vol. 6, no. 2, pp. 28-33, 1959.

[14] T. Furukawa and E. Shimemura, "Predictive control for systems with time delay," International Journal of Control, vol. 37, no. 2, pp. 399-412, 1983.
[15] A. Z. Manitius and A. W. Olbrot, "Finite spectrum assignment problem for systems with delays," Institute of Electrical and Electronics Engineers. Transactions on Automatic Control, vol. 24, no. 4, pp. 541-553, 1979.

[16] W. Michiels, S. Mondié, D. Roose, and M. Dambrine, "The effect of approximating distributed delay control laws on stability," in Advances in Time-Delay Systems, vol. 38 of Lecture Notes in Computer Science and Engineering, pp. 207-222, Springer, Berlin, Germany, 2004.

[17] V. L. Kharitonov, "Predictor-based controls: the implementation problem," Differential Equations, vol. 51, no. 13, pp. 16751682, 2015.

[18] T. G. Molnar and T. Insperger, "On the robust stabilizability of unstable systems with feedback delay by finite spectrum assignment," Journal of Vibration and Control, vol. 22, no. 3, pp. 649-661, 2016.

[19] S. Mondié and W. Michiels, "Finite spectrum assignment of unstable time-delay systems with a safe implementation," IEEE Transactions on Automatic Control, vol. 48, no. 12, pp. 22072212, 2003.

[20] F. Léonard and G. Abba, "Robustness and safe sampling of distributed-delay control laws for unstable delayed systems," IEEE Transactions on Automatic Control, vol. 57, no. 6, pp. 15211526, 2012.

[21] M. Basin and J. Rodriguez-Gonzalez, "Optimal control for linear systems with multiple time delays in control input," IEEE Transactions on Automatic Control, vol. 51, no. 1, pp. 91-97, 2006.

[22] M. Basin, J. Rodriguez-Gonzalez, and R. Martinez-Zuniga, "Optimal control for linear systems with time delay in control input," Journal of the Franklin Institute, vol. 341, no. 3, pp. 267278, 2004.

[23] H. Zhang, G. Duan, and L. Xie, "Linear quadratic regulation for linear time-varying systems with multiple input delays," Automatica, vol. 42, no. 9, pp. 1465-1476, 2006.

[24] Y. Zhou and Z. Wang, "Optimal feedback control for linear systems with input delays revisited," Journal of Optimization Theory and Applications, vol. 163, no. 3, pp. 989-1017, 2014.

[25] B. Zhou, "Input delay compensation of linear systems with both state and input delays by nested prediction," Automatica, vol. 50, no. 5, pp. 1434-1443, 2014.

[26] B. Zhou, "Input delay compensation of linear systems with both state and input delays by adding integrators," Systems \& Control Letters, vol. 82, pp. 51-63, 2015.

[27] S. Xu, J. Lam, B. Zhang, and Y. Zou, "A new result on the delay-dependent stability of discrete systems with time-varying delays," International Journal of Robust and Nonlinear Control, vol. 24, no. 16, pp. 2512-2521, 2014.

[28] J. Diblík, M. Fečkan, and M. Pospíšil, "On the new control functions for linear discrete delay systems," SIAM Journal on Control and Optimization, vol. 52, no. 3, pp. 1745-1760, 2014.

[29] F. Liao and H. Xu, "Application of the preview control method to the optimal tracking control problem for continuous-time systems with time-delay," Mathematical Problems in Engineering, vol. 2015, Article ID 423580, 8 pages, 2015.

[30] Y.-J. Xu and F.-C. Liao, "Preview control for a class of timevarying discrete systems with input time-delay," Control and Decision, vol. 28, no. 3, pp. 466-470, 2013. 
[31] M. Cao and F. Liao, "Design of an optimal preview controller for linear discrete-time descriptor systems with state delay," International Journal of Systems Science. Principles and Applications of Systems and Integration, vol. 46, no. 5, pp. 932-943, 2015.

[32] S.-Y. Han, G.-Y. Tang, and C.-M. Zhang, "Near-optimal tracking control for discrete-time systems with delayed input," International Journal of Control, Automation and Systems, vol. 8, no. 6, pp. 1330-1335, 2010.

[33] M. Athans, "On the design of P-I-D controllers using optimal linear regulator theory," Automatica, vol. 7, no. 5, pp. 643-647, 1971.

[34] F. C. Liao, T. Egami, and T. Tsuchiya, "A general frequency dependent digital optimal preview servo system," Applied Mathematics and Mechanics, vol. 17, no. 4, pp. 319-331, 1996. 


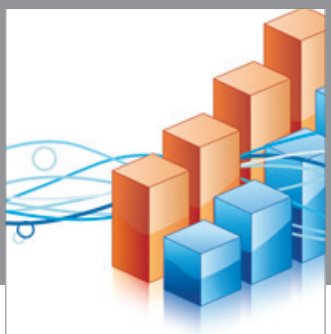

Advances in

Operations Research

vatem alat4

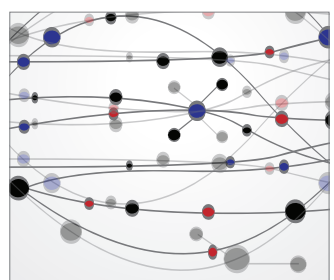

\section{The Scientific} World Journal
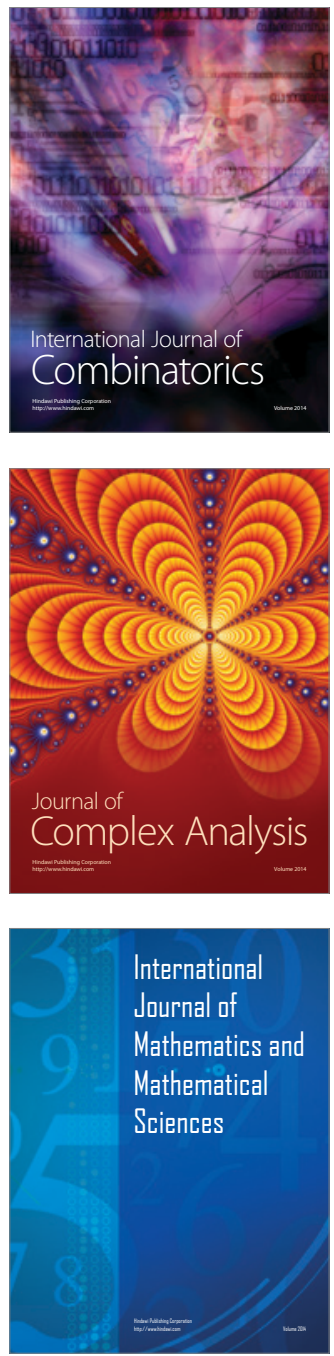
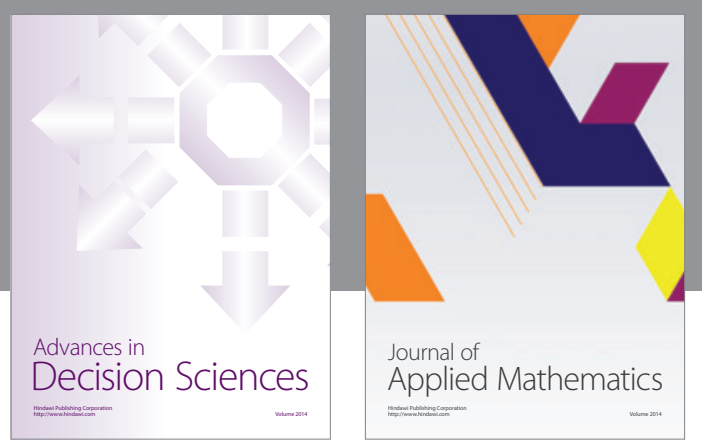

Algebra

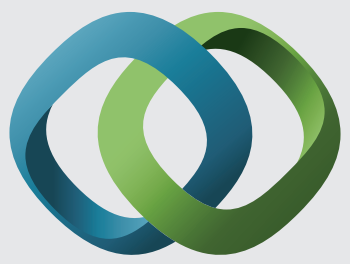

\section{Hindawi}

Submit your manuscripts at

http://www.hindawi.com
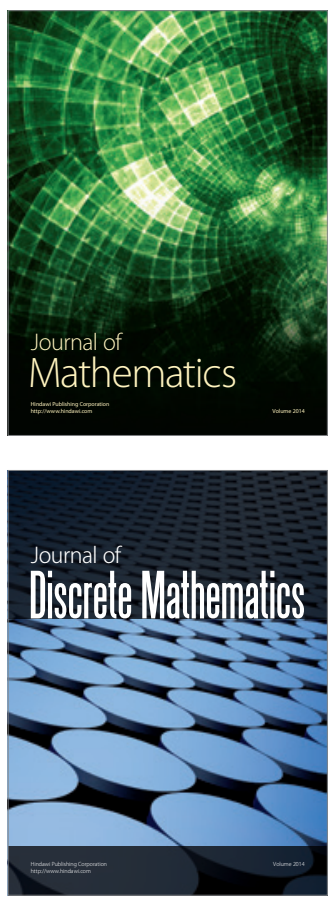

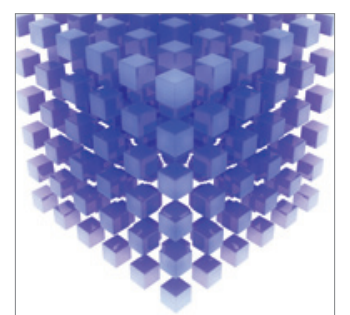

Mathematical Problems in Engineering
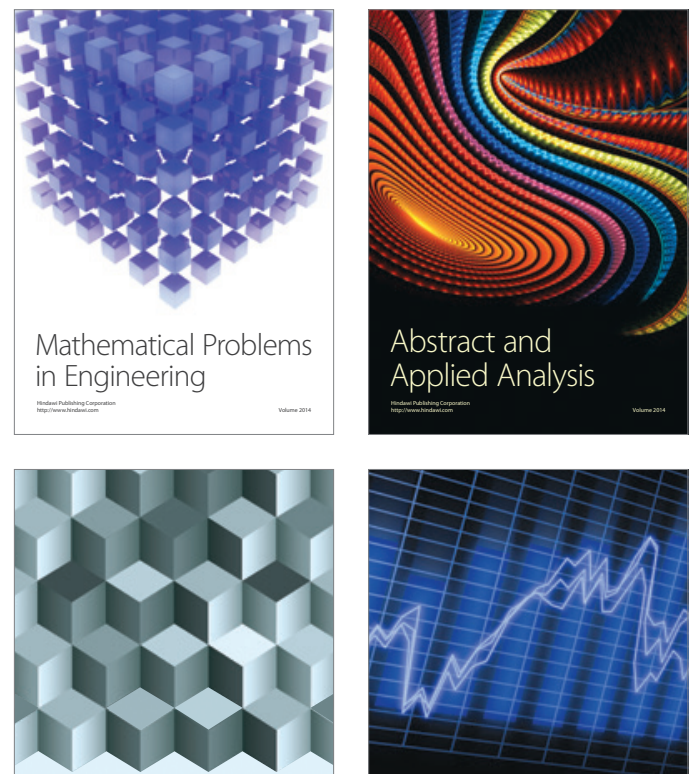

Journal of

Function Spaces

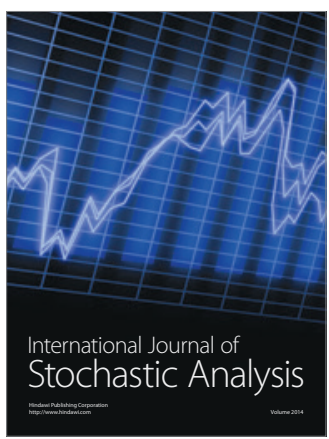

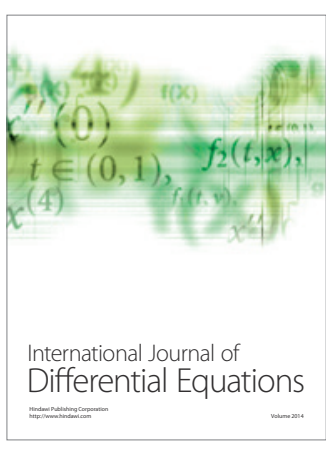
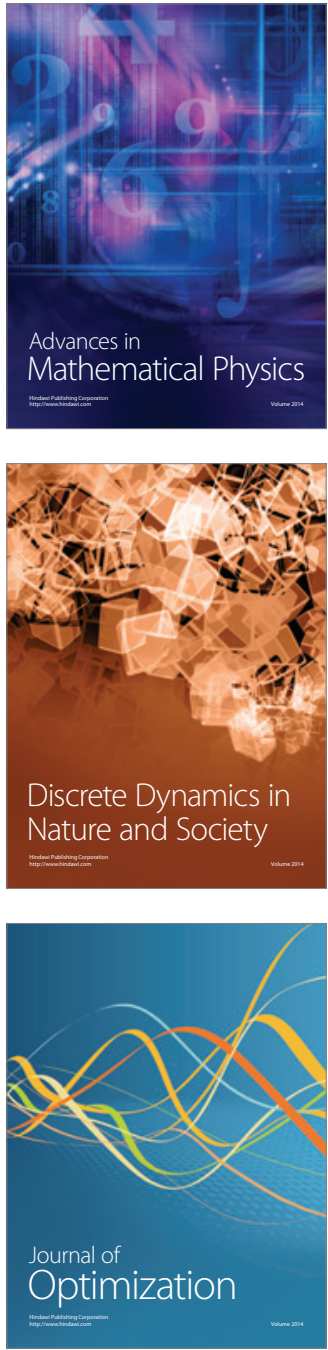